Journal of Applied Pharmaceutical Science Vol. 6 (09), pp. 227-236, September, 2016

Available online at http://www.japsonline.com

DOI: $10.7324 / J A P S .2016 .60934$

ISSN 2231-3354 (cc) BY-NC-SA

\title{
Pharmacotherapy of Opportunistic Infections in HIV Patients: A Review
}

\author{
Sadhana Nittur Holla, Meena Kumari Kamal Kishore, Mohan Babu Amberkar* \\ Department of Pharmacology, Kasturba Medical College, Manipal University, Manipal-576104, Karnataka, India.
}

\begin{tabular}{|c|c|}
\hline ARTICLE INFO & ABSTRACT \\
\hline $\begin{array}{l}\text { Article history: } \\
\text { Received on: } 22 / 12 / 2015 \\
\text { Revised on: } 11 / 03 / 2016 \\
\text { Accepted on: } 17 / 06 / 2016 \\
\text { Available online: } 26 / 09 / 2016\end{array}$ & $\begin{array}{l}\text { With the introduction of antiretroviral therapy, there is a dramatic decrease in the morbidity and mortality } \\
\text { related to human immunodeficiency virus (HIV) infections. Due to varying degree of immunosuppression, } \\
\text { patients are prone to various number of infections in their lifetime. Respiratory system, central nervous system, } \\
\text { gastrointestinal tract and the skin are the most susceptible for opportunistic infections. Timely management of } \\
\text { these microbial infections followed by chemoprophylaxis is essential as per the guidelines for the management }\end{array}$ \\
\hline $\begin{array}{l}\text { Key words: } \\
\text { AIDS, immunosuppression, } \\
\text { antimicrobial therapy, IRIS, } \\
\text { prophylaxis, drug } \\
\text { interactions. }\end{array}$ & $\begin{array}{l}\text { with HIV has always remained a clinical challenge. Consideration is given to the numerous pharmacokinetic } \\
\text { interactions between drugs used to treat and prevent opportunistic infections and antiretroviral drugs, along with } \\
\text { complications like immune reconstitution inflammatory syndrome. Clinicians must be aware about optimal } \\
\text { strategies present for diagnosis, prevention and treatment of opportunistic infections in order to improve health } \\
\text { and provide high quality of care for the patients. }\end{array}$ \\
\hline
\end{tabular}

\section{INTRODUCTION}

Human immunodeficiency virus (HIV) infection accounts for the highest number of death attributable to any single infective agent with a global incidence of 35 million HIV infected people. After the discovery of first case in 1981, India now is the world's third highest country with HIV cases with an estimated incidence of 2.3 million infected people (Park, 2015). The prevalence of HIV in adults is $0.27 \%$, with higher number of men affected than women. In the past decade, the occurrence of new infections in India has reduced by $57 \%$ due to implementation of preventive strategies. The main focus of National AIDS Control Organization (NACO) programme was to prevent the uninfected and treat the infected. Even though widespread surveillance and program has resulted in controlling

* Corresponding Author

Amberker Mohan Babu V, Associate professor, Department of

Pharmacology, Kasturba Medical College, Manipal University,

Manipal-576104, Karnataka, India.

Email:drmohan7amberkar[at]gmail.com newer infections, pharmacotherapy of HIV and concomitant infections is always a challenge (NACO, 2013-14). Diagnosis of opportunistic infection (OI) guides diagnosis of HIV in patients unaware of their positive status. In some, antiretroviral therapy (ART) is delayed due to social stigma or psychological problems, even when informed about positive HIV status. Whereas in some, ART do not help in attaining virological or immunological response due to problems like poor adherence, negligence, financial problems and unexplained biological factors. Pharmacotherapy of opportunistic infections in these group of patients play a major role. Widespread use of highly active antiretroviral treatment (HAART) regimes resulted in substantial and dramatic decrease in acquired immunodeficiency syndrome (AIDS) related opportunistic infection and death in adult and children (AIDS info, 2015). Around 0.5 million lives have been saved due to ART. However, it allows people to live for a longer period, with increased propensity to acquire more opportunistic infections (UNAids, 2014). Appropriate management of OI is as important as ART in preventing morbidity and mortality of HIV patients especially in resource poor countries like India. 
Available guidelines provide literature on management of opportunistic infection, which differ from country to country. The profile of opportunistic infections are changing with increase in incidence of diseases caused by Pneumocystis pneumoniae, Mycobacterium avium complex (MAC) due to better diagnostic methods. In addition, there is an important role of antiretroviral therapy in prevention of OI and of equal concern is, initiation of antiretroviral therapy during treatment of acute OI (Kumarasamy et al., 2011).

\section{WHAT ARE OPPORTUNISTIC INFECTIONS?}

"Infections that cause disease with increased frequency and/or of increased severity among HIV-infected persons, because of immunosuppression" (Kaplan et al., 1996). These infections and associated complications remain an important predictor of hospitalisation and death among HIV patients. In India, 51\% of death among HIV infected are due to opportunistic infections. Incidence depends on the level of immunosuppression and endemic prevalence of the organism in the community. The pattern of symptom presentation varies, with individuals on one side showing positivity for viral load without any manifestations, rest with varying degree of immunosuppression presenting with minor infections to severe life threatening OI and neoplasms (NACO, 2007). The rate of progression of an individual with HIV infection to AIDS depends upon number of viral and host defense factors like health status of the patient, risk behavior, type and frequency of exposure for OIs, adherence to ART and accessibility to drugs for prevention and management of opportunistic infections (AlJabri, 2007).

The primary immuno-pathogenic lesion in HIV infection involves CD4 $\mathrm{T}$ lymphocytes, with quantitative and qualitative defect affecting every aspect of immune system. AIDS defining illness is the occurrence of life threatening OI, malignancies, neurologic diseases in patients with HIV infection and CD4 count $<200$ cells $/ \mu \mathrm{L}$. CD 4 count is the strongest predictor of subsequent disease progression and survival. CD4 counts are checked every 36 months to determine initiation of anti-retroviral therapy, to monitor the immunologic response to therapy and for initiation or discontinuation of prophylaxis for OIs (Hoffman et al., 2010). It has been observed that most of the OIs like cryptosporidiosis, toxoplasmosis, cryptococcal meningitis, pneumocystis pneumonia, histoplasmosis and cytomegalovirus (CMV) retinitis are seen in patients with CD4 $\mathrm{T}$ cells $<200$ cells $/ \mu \mathrm{l}$. Tuberculosis and candidiasis can occur at any CD4 count but usually occurs at counts below 500 cells/ $\mu \mathrm{L}$ (Jung and Pauww, 1998).

\section{ROLE OF ANTI-RETROVIRAL THERAPY}

Anti-retroviral therapy in HIV infection is known to raise CD4 count and reduce plasma HIV ribonucleic acid (RNA) load. ART can restore immunity in the patient and reduce the risk of acquiring new infection. In an acute illness it can prevent progression of HIV to AIDS related illness, improve wellbeing and quality of life. Dilemma is when to start? Earlier ART was considered as the mainstay in managing acute OI, before antimicrobial therapy. Since initiation of ART in a setup of acute OI has been associated with advantages and disadvantages, there are numerous discussions on this issue. Initiation of ART has been effective for those conditions where effective therapy does not exist. It also reduces the chances of developing another OI. An important disadvantage are low plasma levels of drug concentrations due to poor absorption, leading to drug resistance (Kaplan et al., 2009). Development of immune reconstitution inflammatory syndrome (IRIS), pharmacological drug interactions, high pill burden and co-drug toxicities are other few problems faced with ART (Lawn et al., 2007; Kumarasamy et al., 2011). Thus, it is always better to treat or stabilize an opportunistic infection with antimicrobial therapy rather than initiating ART, especially in the setting of acute pneumocystis infection, oesophageal candidiasis and cryptococcal meningitis. However with few exceptions like MAC infection, cryptosporidiosis and microsporidiosis when no specific drug therapy exists, managing with ART becomes the mainstay (NACO, 2013). In such conditions benefits of early therapy with ART outweigh the risk related to the problems of ART (Kaplan et al., 2009). Few factors need to be looked into while managing an opportunistic infection like degree of immunosuppression, problems with ART, availability of effective therapy for the OI and willingness of the patient to adhere to the drug therapy. OI can alter the metabolic and excretory function which may alter distribution or clearance of the drugs thus predisposing to toxicity. Acute gastrointestinal OIs can decrease absorption of antiretroviral drugs, leading to inefficiency or development of resistance (Brooks et al., 2009).

\section{IMMUNE RECONSTITUTION INFLAMMATORY SYNDROME}

Although ART restores immune function with increase in CD4 count and reduction in plasma HIV RNA load, it is not free of complications especially in the first 6 months. HIV associated IRIS is important clinical condition posing a major threat in the management of OI. It is the "occurrence or manifestations of new OIs or existing OIs within six weeks to six months after initiating ART; with an increase in CD4 count." It is an atypical presentation of OI with different patterns of manifestation. "Paradoxical IRIS", where a known opportunistic infection being treated, recur or worsens in spite of favorable response to therapy prior to ART. "Unmasking IRIS" is when an existing infection in the body which was latent or subclinical, becomes active with restoration of an immune response after starting anti-retroviral therapy (Sharma and Soneja, 2011). However, after 12 weeks of ART initiation, occurrence of new OIs are less likely due to IRIS. It could represent incomplete immunity or ART failure predisposing for a newer OI (Kaplan et al., 2009). The incidence keeps varying depending upon the population and prevalence of infecting organism in the community. Over all incidence in India is 35\% with mucocutaneous infections, tuberculosis and pneumocystis 
pneumonia being the most common infections associated with IRIS. Risk is higher in patients who are started on ART for the first time, who have low CD4 count below 50 cells $/ \mu \mathrm{L}$ prior to the initiation of ART and shorter duration of treatment for OI. IRIS are managed with anti-inflammatory agents like non-steroidal antiinflammatory drugs (NSAIDs) and corticosteroids (Walker et al., 2015). Considering the situation in India where burden of opportunistic infections is large and increased enrolment of ART, development of IRIS is certain. So guidelines stresses on treating and stabilizing OI, followed by managing with ART (NACO, 2013).

\section{ANTIMICROBIAL THERAPY}

Opportunistic infection continues to be one of the most universal complications in HIV infection. The life span of an HIV infected individual is shortened as such by these acquiring infections than the virus itself. Appropriate and timely prophylaxis of opportunistic infections may decrease the incidence and reduce the financial burden especially in resource poor countries like India. Early diagnosis and clinical management must be a part of routine care (Saha et al., 2011). Antimicrobial therapy is indicated for patients who are on HIV management with ART and timely diagnosed with opportunistic infection, for patients who are on ART but do not attain virological or immunological response and for management in paediatric age group where OI is the indicator of the disease. ART is deferred till 15 years of age due to drug toxicity and resistance (NACO, 2006). Another significant aspect is appropriate intervention of an OI may reduce the transmission of microbial infections to others in the community.

\section{HIV AND RESPIRATORY SYSTEM}

Respiratory system is the most susceptible system to pathogens. It can be milder upper respiratory tract infection to severe life threatening pneumonia causing increased morbidity and mortality in HIV. Common organisms are Pneumocystis jiroveci, MAC and bacterial agents causing pneumonia. More than one pathogen may be involved, where diagnosis becomes an essential criteria. However majority of the cases can be effectively prevented with timely prophylaxis and management with antimicrobial agents.

\section{Pneumocystis jiroveci pneumonia (PJP)}

Pneumocystis jiroveci is a fungus common in patients with HIV infection with an incidence of $20-30 \%$. They may be presenting manifestation in patients who have not yet been diagnosed with HIV. Around $80 \%$ of healthy children have antibodies to Pneumocysti jiroveci by 2-4 years of age. Adult pneumonia results from re-activation of latent infection or new exposure to the organism transmitted by inhalational route. Infection occurs when CD 4 count falls below 250 cells $/ \mu \mathrm{L}$. They have a nonspecific onset with fever, dry cough and progressing to severe dyspnoea and pneumothorax (Wazir and Ansari, 2004). Patients with PJP require 3 weeks of treatment, compared to two weeks therapy in non HIV individuals. Hospital admissions with parenteral co-trimoxazole and oxygen therapy are required till they improve and can absorb oral medications. Mild disease can be treated with oral therapy on an out-patient basis (Wilkin and Feinberg, 1999). They respond to co-trimoxazole (SMX-TMP), recommended for treatment of mild, moderate or severe PJP. [Table 1] Problems foreseen are bone marrow suppression with trimethoprim and hypersensitivity reaction with sulfamethoxazole. Desensitisation regimen is followed after mild reaction with an interval of 2 weeks of temporary drug stoppage. About $70 \%$ of individual respond to desensitisation. Alternative regimens include trimethoprim plus dapsone, intravenous pentamidine, clindamycin plus primaquine and atovaquone suspension. The need of an alternative drug prevails when severe adverse reactions develop, if drug is contraindicated or if there is no response to treatment. Corticosteroids are used as adjunctive therapy in severe cases (NACO, 2007; Vilar et al., 1999). Initiation of ART within 2 weeks of diagnosing PJP infection resulted in major complications such as IRIS and drug toxicities (Kaplan et al., 2009). So, it is essential to stabilize pneumonia with antimicrobial therapy for 2 weeks or till patient responds, then starting or switching over to anti-retroviral management (NACO, 2013). Routine prophylaxis with cotrimoxazole is recommended under NACO programme. It is effective in preventing PJP, toxoplasmosis, nocardiosis and diarrhoea causing organisms like isosporiasis (Castro and Morrison-Byrant, 2010).

\section{Mycobacterium avium complex}

Among the non-tubercular mycobacterial infections, MAC is most commonly seen in immunocompromised individuals when CD4 cell count is less than 50 cells $/ \mu \mathrm{L}$. It is difficult to get the exact rates of incidence and prevalence, as isolation of organism does not indicate the presence of active infection. They have a subtle course in immunocompetent individuals, but produce severe life threatening disseminated disease in AIDS patients. They are transmitted by inhalation, ingestion or inoculation route. Symptoms are nonspecific initially and progress later to develop multi-organ dysfunction. Severe MAC syndrome is associated with persistent fever, night sweats, diarrhoea, pulmonary infiltrates, hepatomegaly and anaemia (Johnson and Odell, 2014).

Clarithromycin is the cornerstone of MAC therapy. An excellent substitute is azithromycin which bears advantage over clarithromycin in terms of drug interactions or liver dysfunction [Table 1]. Combination therapy with ethambutol is preferred for effective clearance of organism and to prevent emergence of drug resistance. Rifabutin is added when disease is advanced with high mycobacterial load or when ART is not available. Rifabutin is preferred over rifampicin as it is devoid of drug interactions with drugs like protease inhibitors (PI) and non-nucleoside reverse transcriptase inhibitors (NNRTI). The duration of therapy is lifelong, however it can be discontinued after 12-14 months if patient is asymptomatic and CD 4 count stays $>100$ cells $/ \mu L$. Due to prolonged therapy signs of drug toxicity like blurring of vision, change in colour perception, signs of neurotoxicity and 
hepatotoxicity must be watched for. Injectable aminoglycoside like streptomycin/amikacin or flouroquinolone like levofloxacin may be required when treatment fails after 4 weeks (Seddon and Bhagani, 2011).

In an acute setting of MAC infection, ART stabilizes the patient and reduces nonspecific symptoms (NACO, 2013). Prophylaxis is indicated when CD4 count falls below 50 cells $/ \mu \mathrm{L}$ or in disseminated mycobacterial infection with clarithromycin or azithromycin. Patient compliance is better with azithromycin as the dose is once in a week (NACO, 2007). Azithromycin protects against disseminated MAC in patients not responding to ART and has no role in the prevention of IRIS due to MAC (Phillips et al., 2002).

\section{Pneumonia}

As discussed before respiratory tract is the most frequent site for repeated infections in both immunocompetant and immunosuppressed individuals. If unnoticed or not treated with appropriate antibiotics they produce severe life threatening complications. Most common organisms are Streptococcus pneumoniae, Haemophilus influenzae, Pseudomonas aeruginosa and Staphylococcus aureus. Organisms causing atypical pneumonia are Legionella pneumophila, Mycoplasma pneumonia and Chlamydia species (Benito et al., 2012). Clinical manifestations are similar to non HIV infected individuals; however they are always in severe and disseminated form with bacteraemia, empyema, sepsis, drop in oxygen saturation and death.

Moderate to severe pneumonia needs admission and management with oxygen, parenteral antibiotics and supportive care. Milder forms are treated on an outpatient basis. Antibiotic regimen selected to treat pneumonia are similar to that treated for non HIV infected individual. Beta lactams are preferred and macrolides are less prescribed due to resistance. Respiratory fluoroquinolones are alternative if allergic to penicillin group of antibiotics. Quinolones are also active against mycobacterium tuberculosis which may cause delay in diagnosis, treatment and development of resistant strains (NACO 2007; Huang et al., 2007). A decision to change the drug regimen arises when no improvement in clinical, radiological or microbiological parameters occurs. Severe pneumonia is treated with parenteral antibiotics, analgesics, antipyretics, oxygen inhalation, chest physiotherapy and nutritional support. Once pneumonia is stabilized and antibiotic therapy is completed, ART is given (NACO, 2013). Routine prophylaxis with antibiotics is not recommended, as the sensitivity to antibiotics will be a lost which becomes a major hurdle to treat an acute attack. Long term therapy also leads to drug toxicity. When CD4 counts are $\leq 200$ cells $/ \mu \mathrm{L}$, vaccination against Streptococcus pneumoniae can be employed. Single dose of 23-valent polysaccharide pneumococcal vaccine is given if not vaccinated in the last five years. Annual influenza vaccination are also given (Davis et al., 2008).

\section{HIV AND GASTROINTESTINAL SYSTEM}

Gastrointestinal system is most accessible and the next common system to be involved in HIV infection with more than $90 \%$ incidence. The extent of involvement depends on the CD4 count, degree of immunosuppression, causative organism and duration of disease. Spectrum of lesions ranges from oral and oesophageal candidiasis, viral lesions of oral cavity caused by Epstein-Barr virus (EBV), Herpes simplex virus (HSV) and CMV, small and large bowel diarrhoea, pancreatitis and anorectal lesions. For infections where specific and effective drugs are available such as Candida infection and cryptosporidiosis, they are managed initially with anti-microbial agents. For virus causing oral ulcers do not have a specific curative agent, ART is started initially. Most of the time effective drug may not be available (Al Anazi, 2009). Diagnosis, clinical evaluation and laboratory confirmation becomes an essential factor in identifying the causative organism as set up and management for each infections are different.

\section{Candidiasis}

Infection with Candida produces mucocutaneous lesions in immunocompromised individuals. They affect mucosal lining of mouth, throat, oesophagus, intestine and vagina. Candida infection occurs regardless of CD4 count. Intense involvement and invasive lesions are seen with HIV infection when CD4 count drops below 200 cells $/ \mu \mathrm{L}$. Causative organisms are Candida albicans and Candida glabrata. Candida lesions are white patches in the oral cavity and oesophagus producing maximum discomfort and painful swallowing. There is loss of appetite and weight, anaemia, muscle wasting and poor immunity (Badiee and Hashemizadeh, 2014).

Oral fluconazole is an effective therapy for control and treating candidiasis. [Table 1] Most of the patients respond to single course of $150 \mathrm{mg}$ tab 1-2 tablets per day. It is potent CYP3A4 inhibitor and demonstrates number of drug interactions with ritonavir, NNRTI, clarithromycin and rifampicin. Topical agents with clotrimazole troche and nystatin oral suspension therapy are indicated in mild disease, when azoles are not tolerated or contraindicated in renal failure or avoided due to drug interactions. Oesophageal candidiasis is treated with fluconazole at a higher dose of $400 \mathrm{mg} /$ day or a second generation itraconazole oral solution. The duration of treatment is not more than 21 days. If patient does not respond even after 3 weeks, diagnosis is reconfirmed. For relapse cases, newer antifungal azole agents like voriconazole and posaconazole may be required (Greenspan, 1994).

Voriconazole is associated with more adverse events when compared to fluconazole, so use is restricted only to severe disseminated candidiasis (Pappas et al., 2004). Once the lesion resolves and patients are able to take oral medications, antiretroviral therapy is given. Primary or secondary prophylaxis is not indicated unless it is a recurring infection. However it should be discontinued after CD4 count rises above 200 cells $/ \mu \mathrm{L}$. 


\section{Oral hairy leukoplakia (OHL)}

It is a non-malignant condition caused by Ebstein Barr virus involving the oral cavity with white hairy patches over the tongue and palate. Infection can occur in healthy individuals, but more common in immunocompromised individual with incidence $50 \%$ in HIV disease. It has an important place in the clinical management as the oral plaques resemble oral candidiasis. Diagnosis is essential, as management varies between the two.

Usually OHL does not require management. Clinically it can be identified by scraping the lesions; they do not separate from the mucosal surfaces. Diagnosis is confirmed by determining EBV antigens from the scraped samples (Kreuter and Wieland, 2011). Topical application with podophyllin resin and tretinoin twice daily for 3 weeks is effective [Table 1]. Temporary disappearance of the lesion can be achieved by oral acyclovir therapy for 2-3 weeks (Brasileiro et al., 2014).

\section{Cryptosporidiosis}

A protozoan infection caused by Cryptosporidium parvum affects the small bowel mucosa, causes diarrhoea in HIV individuals in the developing countries. Cryptosporidiosis may be the first AIDS defining illness occurring when CD4 count falls below 100 cells $/ \mu \mathrm{L}$. Infection may be asymptomatic or cause severe profuse watery diarrhoea. The incidence of this infection has decreased in the developed world due to the use of antiretroviral therapy (Ajjampur, 2007).

Rehydration is mainstay of therapy. Nitazoxanide a broad spectrum anti-parasitic drug is effective against the protozoa and is approved for the treatment. It causes $90 \%$ clearance of parasitic growth in cell culture and $95 \%$ reduction of oocysts in stools (Kadappu et al., 2002). Azithromycin is safe and effective against cryptosporidium infection. Short course therapy for a week is helpful in symptomatic control and extended course for 2 weeks clears Cryptosporidium parvum oocysts from stool (Cabada and White Jr, 2010). Paromomycin and dapsone are the alternative drugs [Table 1]. Anti-motility agents and nutritional supplementation are the supportive measures. Meta-analysis of numerous published studies have revealed that paramomycin has poor relapse rate and no more effective than placebo in reducing frequency of loose stools. Since the effectiveness of a specific agents are doubtful in controlling diarrhoea, early ART is ideal for effective cure. ART causes restoration of immune response with complete resolution of clinical symptoms (Kaplan et al., 2009).

\section{HIV AND NERVOUS SYSTEM}

Advanced HIV infection causes opportunistic infection of brain and spinal cord, which is independent of CD4 count. Most common organisms are Cryptococcus neoformans, Toxoplasma gondii and Cytomegalovirus. Less common ones are Mycobacterium tuberculosis, Streptococcus pneumoniae and Neisseria meningitidis. Organisms may infect the meninges or present as a focal neurological lesion with headache, convulsions and raised intracranial pressure. Diffuse cerebral and spinal involvement may lead to encephalitis and myelitis (Tan et al., 2012).

\section{Cryptococcal meningitis}

Cryptococcus species are one of the most important causes of morbidity and mortality in immunocompromised individual. Occurs in $10 \%$ of HIV individuals and when CD4 count falls below 100 cells/ $\mu \mathrm{L}$. Initially produce signs of meningeal irritation and may progress to cause encephalitis. Organisms also produces skin and pulmonary disease. It is a fatal unless treated (Antinori, 2013).

The treatment is split into three phases. [Table 1] Initial induction phase for 2 weeks with amphotericin-B and 5flucytosine, combining these two antifungal decreases the burden of budding yeast cells in CSF and has a better cure rate than administering amphotericin alone. This is followed by consolidation phase for 8 weeks with fluconazole or itraconazole alternatively until CSF becomes sterile. This approach clears $70 \%$ of the fungus and $10 \%$ reduction in mortality. The third phase is the lifelong maintenance therapy with fluconazole to prevent relapses. Electrolytes, renal parameters and liver function tests are monitored periodically. Voriconazole is given, if fluconazole is not tolerated (Saag et al., 2000).

Survival rate of patients with cryptococcal meningitis has dramatically improved with ART, but initiating ART in acute infection may flare up the scenario causing fatal IRIS. Few studies conducted to assess the timing of ART in acute cryptococcal meningitis with concurrent HIV infection reveals no difference in mortality rates between the groups with early or late initiation of ART (Njei et al., 2013). However to reduce the chances of IRIS, early management with antifungals for 2 weeks followed by ART is recommended (NACO, 2013).

\section{Cerebral toxoplasmosis}

Toxoplasma gondii causes toxic encephalitis in HIV infected individuals producing symptoms of headache, confusion, seizures and motor weakness. Infection is acquired by inhalation of oocysts in early childhood days, but are asymptomatic. Reactivation of infection with CNS, pulmonary and retinal manifestation occurs due to immunosuppression when CD4 counts are below 100 cells/ $\mu \mathrm{L}$ (Saadatnia and Golkar, 2012).

A combination of pyrimethamine and sulfadiazine with leucovorin is given for 6 weeks [Table 1]. They penetrate the CNS in the absence of inflammation. Folinic acid is added to the regimen to reduce haematological side effects of pyrimethamine. Skin rashes, crystal nephropathy due to sulfadiazine may lead to discontinuation of sulfadiazine (Jayawardena, 2008).

Alternative treatment with clindamycin and pyrimethamine is equally effective and applicable for patients allergic and intolerant to sulfa drugs. High dose of clindamycin alone in resource poor countries brings about radiological and clinical cure (Madi et al., 2012). Other agents used for treatment include parenteral TMP-SMX for severely ill patients and atovaquone combination with sulphadiazine or pyrimethamine. 
They are effective, but research studies and clinical experience is limited (Seddon and Bhagani, 2011).

Maintenance therapy with the above drugs in low doses are given lifelong to prevent recurrences or till CD4 count raises and stays above 200 cells/ $\mu \mathrm{L}$ for 6 months. Toxoplasmosis have been reported in cases with high CD4 count above 200 cells $/ \mu \mathrm{L}$, where cotrimoxazole has been successfully used to cure the lesions (Patil et al., 2007) (Swami et al., 2015). Dexamethasone reduces oedema and inflammation; however it can precipitate other opportunistic infections. ART is delayed upto 6 weeks after stabilizing the patient with antibiotics and conservative management (NACO, 2013).

\section{Cytomegalovirus}

Human cytomegalovirus infection is common with a prevalence of $40-70 \%$ in HIV adults, occurring due to reactivation of the latent infection. It causes localized organ damage or disseminated disease when CD 4 count fall below 100 cells $/ \mu \mathrm{L}$. It affects the eye, lung, CNS and colon. CMV retinitis is most common with an incidence of $30 \%$ followed by colitis, esophagitis, pneumonitis and encephalitis. Unilateral or bilateral retinitis presents as peripheral visual defects with floaters and scotoma. They progress to cause defect in central vision involving optic nerve and macula and ultimately causing retinal detachment and blindness (Kirubakaran, 2004).

Early treatment is the mainstay in preventing further progression and complications [Table 1]. Ganciclovir is the drug of choice for CMV disease. However it can damage the precursor cells in the bone marrow and can produce neuropsychiatric changes. Valaganciclovir an oral agent for CMV is a prodrug with higher oral bioavailability, better efficacy but safety profile is similar to ganciclovir (Hoglund et al, 2001). To avoid systemic toxicity, ganciclovir implants or intravitreal injection for a period of 6 months were tried, but were unsuccessful due to retinal detachment and recurrences (Seddon and Bhagani, 2011). Ganciclovir prophylaxis is required for the contralateral eye and to prevent relapse. Foscarnet, cidofovir and formivirsen are given in failed ganciclovir therapy or in drug resistance. Intravitreal route are also available (Boeckh and Geballe, 2011). In patients with acute retinitis, flaring up of uveitis and blindness occurs due to immune reconstitution due to anti-retroviral therapy. Controlling acute infection with anti-viral therapy for 2-3 weeks then starting ART therapy decreases the incidence of immune reconstitution uveitis. However if there is no specific therapy or if there is potential risk of developing other OI, ART can be started early (NACO, 2013).

\section{HIV AND SKIN}

Occurrence of dermatological infections are irrespective of CD4 count and seen at all stages of immunosuppression reactions. Multiple bacterial organisms are involved and cause infections like pustules, furuncles, impetigo and deep abscess.
Management is similar to non-HIV infected people with antibiotic therapy and surgical drainage. Fungal infections like dermatophytosis, seborrheic dermatitis and mycosis are rare but respond well to antifungal treatment. Herpes simplex, herpes zoster and human papilloma virus have a recalcitrant course in HIV infected. Diagnosis is crucial. Cure rates are poor because of deeper, mixed and resistant infections. Anti-retroviral therapy can be initiated early and may respond well (Creed R et al., 2009).

\section{Herpes simplex virus}

Herpes simplex virus has a severe and disseminating course in HIV infected people with chronic painful vesicular eruptions and ulcers in the skin and mucous membrane of oral and anogenital region. They may be acquired as primary infection or reactivation of a latent subclinical infection. Genital ulcerations are a risk factor for acquiring HSV infection.

They respond well to acyclovir and oral lesions resolve within 10 days, but for genital lesions treatment need to be extended for 15 days. [Table 1] Valacyclovir and famciclovir are alternatives for acyclovir toxicity and resistance (Barnabas and Celum, 2012). Parenteral acyclovir is required in advanced illness, severe mucocutaneous lesion, colitis, pneumonitis and encephalitis. Patients receiving high dose IV acyclovir should be monitored for renal functions once or twice weekly (Kaplan et al., 2009). Following treatment of acute lesions, chronic suppressive therapy can be given for an indefinite period to prevent recurrences or relapses. Treatment period is not depended on CD4 count (Strick et al., 2006).

\section{Herpes zoster virus}

The incidence of herpes zoster in HIV infected person is $8-11 \%$, about $25-30$ times higher than people of general population. Reactivation of dormant varicella zoster virus (VZV) in the nerve roots occurs due to immunosuppression, when CD4 count is below 200 cells/ $\mu \mathrm{L}$ in HIV. Tingling, burning sensation over the dermatome followed by a vesicular rash and intense scarred lesions are observed. The incidence of post herpetic neuralgia is high in HIV infected.

Disseminated infection may lead to retinitis, pneumonitis and encephalitis which requires "extensive" therapy with parenteral acyclovir. Mild to moderate lesions require episodic therapy with oral Acyclovir [Table 1]. Aim of treatment is to reduce pain and prevent complications (Dworkin et al., 2007).

Other alternatives are valacyclovir or famciclovir in case of poor compliance or treatment failure (Mandal, 2006). Analgesics and anti-inflammatory agents might be essential during acute episode, for 'healing and reducing distress of post herpetic neuralgia. A 3-week tapering course of corticosteroids diminishes acute zoster pain, faster healing, reducing need of analgesic therapy and return of daily activities (Harpaz et al., 2008). 
Table 1: Chemotherapy and prophylaxis of different opportunistic infections in HIV infected people.

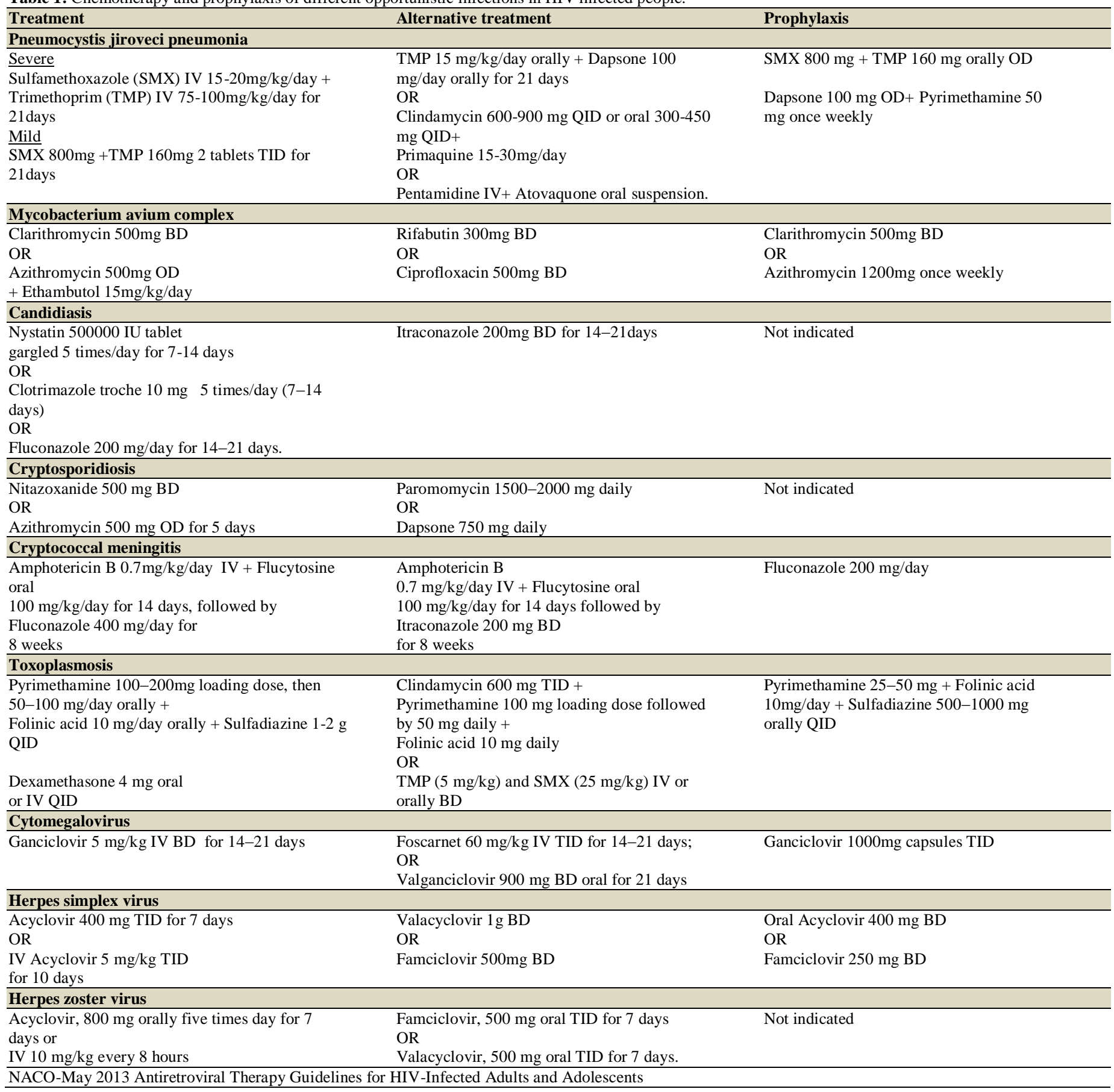

\section{CHEMOPROPHYLAXIS}

Prevention of opportunistic infections in patients with HIV disease is important to optimize outcome. All HIV-related infections and malignancies multiply in frequency as the absolute CD4 T-lymphocyte count falls toward 200 cells $/ \mu \mathrm{L}$. Therefore, estimation of CD4 count is essential and carried out every 3 to 6 months to determine the risk of infection in a specific patient and serves as a guide to drug prophylaxis (Ford, 2015). For those patients who are not or recently started ART, unable to achieve CD4 count above threshold level, virological failure, AIDS defining illness stage 3 or 4 and in case of contact exposure, primary prophylaxis is given to prevent first episode of opportunistic infection.

Secondary prophylaxis is continuation of treatment in low doses for a period till immunity recovers and further to prevent recurrence and relapses. Maintenance phase is continued till CD4 count is above 200 cells $/ \mu \mathrm{L}$ for at least a period of 6 months (NACO, 2007). 


\section{DRUG INTERACTIONS}

Drug-drug interaction is the major hindrance in successful therapy for opportunistic infections in HIV. Interaction occurs between the anti-retroviral drugs and antimicrobial drugs. They induce and inhibit metabolism of each other rendering one or both drugs ineffective or increases the risk of toxicity. Change in the plasma level of drug concentrations occurs by inducing or inhibiting the key enzyme Cytochrome (CYP) P450 monoxygenase (Walubo, 2007). Nucleoside reverse transcriptase inhibitors (NRTIs) of ART are devoid of drug interactions and do not significantly change the drug levels. NNRTIs are substrates as well as inducers of CYP enzymes causing faster elimination of drugs and leading to therapeutic failure. Efavirenz can also inhibit enzyme.

All protease inhibitors are metabolized by CYP3A4 except nelfinavir. Ritonavir is a potent CYP3A4 inhibitor and is used with other PIs to increase their bioavailability. Rest are weak inhibitors of CYP3A4. Azole antifungals are CYP3A4 inhibitors. When used along with PI and other CYP3A4 inhibitors, increased concentration of both the drugs occurs due to bidirectional inhibition. Azoles also inhibits CYP2C9, CYP2C19 and CYP2D6. Clarithromycin is a strong and erythromycin is a weaker inhibitor of CYP3A4.

Though rifampicin is a potent drug for MAC, rifabutin is preferred as it a lesser inducer of CYP3A4 (Dooley et al., 2008). By modifying the dose of drugs and aptly knowing the metabolism and enzymes related to it, we can reduce the occurrence of unwanted interactions and prevent treatment failure. Fixed dose combination of drugs does not allow dose to be altered, in that case it is better to change and substitute with another drug. Selection of new drugs with less drug interaction potential will be helpful. Finally, if feasible therapeutic drug monitoring is advisable (Pham, 2010).

\section{OPPORTUNISTIC INFETIONS IN CHILDREN}

Children are infected with opportunistic infections through placental transmission like CMV, toxoplasmosis or via horizontal spread. In contrast to the adults where most of the opportunistic infections are due to reactivation of latent infections, OI represent primary infection with the pathogen in children. Pharmacotherapy for OIs in children are similar to the adults, but are always challenging in terms of clinical and laboratory diagnosis.

Very often the typical clinical manifestations are absent, with children are unable to describe symptoms. Presence of transplacental crossed antibodies in the serum interfere with diagnosis. Successful implementation and maintenance of HAART, resulting in improved immune status, is the most important factor in control of opportunistic infections among both HIV-infected adults and children. But few issues related to drug pharmacokinetics, formulation, ease of administration, drug dosing and toxicity are important area of concern among children (Mofenson et al., 2014).

\section{OPPORTUNISTIC INFETIONS IN PREGNANCY}

There is no difference in the spectrum of infections and symptoms among the pregnant women when compared to the general population. Physiological changes occurring during pregnancy may affect the pharmacokinetics and diagnosis of OIs. Diagnostic procedures for OI involving radiation exposure should be avoided or limited in pregnant women. If OI is diagnosed and patient not on ART, immediate initiation of ART should be encouraged to minimize the risk of perinatal transmission of HIV. Factors affecting ART in pregnant women with OI include clinical condition, gestational age, viral load, drug toxicities and interactions between ART and OI drugs. Exposure of pregnant women to drugs require detailed anomaly scan by 20 weeks and then followed every 4 weeks to assess foetal growth and development (Nelson et al., 2011).

\section{CONCLUSION}

In order to reduce HIV related morbidity and mortality it is very important to understand that HIV presents as an opportunistic infection. The profile of opportunistic infections varies depending upon the infection prevalent in the community. This highlights the need for early screening and diagnosis in order to improve decisions regarding prophylaxis for prevention and appropriate therapeutic intervention. However, response to therapy depends upon several factors like number of previous episodes and degree of immunodeficiency. Severity of illness is also an essential factor in deciding protocol for management. Prognosis depends upon the drug chosen and timing of initiation of therapy. Comorbidities are also responsible for the important cause of failure in therapy. Thus there is need for screening of patients at risk of opportunistic infection, increasing awareness among health care providers, maximizing benefits from ART, judicious use of chemoprophylaxis and immunoprophylaxis which would prevent various opportunistic infections leading to a favorable impact on the survival of HIV infected people.

Financial support and sponsorship: Nil.

Conflict of Interests: There are no conflicts of interest.

\section{REFERENCES}

AIDSinfo- 2015. Guidelines for Prevention and Treatment of Opportunistic Infections in HIV-Infected Adults and Adolescents [ONLINE] Available from: https://aidsinfo.nih.gov/contentfiles/ lvguidelines/Adult_OI.pdf [Accessed 20 November 2015]

Ajjampur SS, Asirvatham JR, Muthusamy D, Gladstone BP, Abraham OC, Mathai D, et al. Clinical features \& risk factors associated with cryptosporidiosis in HIV infected adults in India. Indian J Med Res, 2007;126(6):553-7.

Al-Anazi AR. Gastrointestinal opportunistic infections in human immunodeficiency virus disease. Saudi J Gastroenterol, 2009; 15(2): 95.

Al-Jabri AA. Mechanisms of host resistance against HIV infection and progression to AIDS. Sultan Qaboos Univ Med J, 2007; $7(2): 82$.

Antinori S. New insights into HIV/AIDS-associated cryptococcosis. ISRN AIDS, 2013; 2013: 471363. doi: 10.1155/2013/471363. 
Badiee P, Hashemizadeh Z. Opportunistic invasive fungal infections: diagnosis \& clinical management. Indian J Med Res, 2014; 139(2): 195

Barnabas RV, Celum C. Infectious co-factors in HIV-1 transmission herpes simplex virus type-2 and HIV-1: new insights and interventions. Curr HIV Res, 2012;10(3):228-37.

Benito N, Moreno A, Miro JM, Torres A. Pulmonary infections in HIV-infected patients: an update in the 21st century. Eur Respir J, 2012;39(3):730-45.

Boeckh M, Geballe AP. Cytomegalovirus: pathogen, paradigm, and puzzle. J Clin Invest, 2011;121(5):1673-80.

Brasileiro CB, Abreu MHN, Mesquita RA. Critical review of topical management of oral hairy leukoplakia. World J Clin Cases, 2014;2(7):253-6.

Brooks JT, Kaplan JE, Masur H. What's new in the 2009 US guidelines for prevention and treatment of opportunistic infections among adults and adolescents with HIV. Top HIV Med, 2009;17(3):109-14.

Cabada MM, White AC Jr. Treatment of cryptosporidiosis: do we know what we think we know? Current Opin Infect Dis, 2010; 23(5):494-9.

Castro JG, Morrison-Bryant M. Management of pneumocystis jirovecii pneumonia in HIV infected patients: current options, challenges and future directions. HIV/AIDS (Auckl), 2010;2:123-34.

Creed R, Morrison LK, Ravanfar P, Mendoza N, Tyring S. Skin complications of HIV infection. Expert Rev Dermatol, 2009; 4(5): $509-21$.

Davis JL, Fei M, Huang L. Respiratory infection complicating HIV infection. Curr Opin Infect Dis, 2008;21(2):184

Dooley KE, Charles F, Andrade AS. Drug interactions involving combination antiretroviral therapy and other anti-infective agents: repercussions for resource-limited countries. J Infect Dis, 2008;198(7):948-61.

Dworkin RH, Johnson RW, Breuer J, Gnann JW, Levin MJ, Backonja $\mathrm{M}$, et al. Recommendations for the management of herpes zoster. Clin Infect Dis, 2007;44(1):1-26.

Ford N, Meintjes G, Pozniak A, Bygrave H, Hill A, Peter T, et al. The future role of $\mathrm{CD} 4$ cell count for monitoring antiretroviral therapy. Lancet Infect Dis, 2015;15(2):241-7.

Greenspan D. Treatment of oropharyngeal candidiasis in HIV positive patients. J Am Acad Dermatol, 1994;31(3):51-5.

Harpaz R, Ortega-Sanchez IR, Seward JF. Prevention of Herpes Zoster- CDC. MMWR, 2008;57:1-30

Hoffman J, Van Griensven J, Colebunders R, McKellar M. Role of the CD4 count in HIV management. HIV Therapy, 2010;4(1):27-39.

Hoglund M, Ljungman P, Weller S. Comparable aciclovir exposures produced by oral valaciclovir and intravenous aciclovir in immunocompromised cancer patients. J Antimicrob Chemother, 2001;47(6):855-61.

Huang L, Cattamanchi A, Davis JL, Boon SD, Kovacs J, Meshnick S, et al. HIV-associated Pneumocystis pneumonia. Proc American Thorac Soc, 2011;8(3):294-300.

Jayawardena S, Singh S, Burzyantseva O, Clarke H. Cerebral toxoplasmosis in adult patients with HIV infection. Hospital Physician, 2008;44(7):17-24.

Johnson MM, Odell JA. Nontuberculous mycobacterial pulmonary infections. J Thorac Dis, 2014;6(3):210-20.

Jung AC, Paauw DS. Diagnosing HIV-Related Disease. J Gen Intern Med, 1998;13(2):131-6.

Kaplan JE, Benson C, Holmes KK, Brooks JT, Pau A, Masur H, et al. Recommendations from CDC, the National Institutes of Health, and the HIV Medicine Association of the Infectious Diseases Society of America. Guidelines for prevention and treatment of opportunistic infections in HIV-infected adults and adolescents. MMWR Recomm Rep, 2009;58(4):1-207.

Kaplan JE, Hu DJ, Holmes KK, Jaffe HW, Masur H, De Cock KM. Preventing opportunistic infections in human immunodeficiency virus-infected persons: implications for the developing world. Am J Trop Med Hyg, 1996;55(1):1-11.
Kadappu KK, Nagaraja MV, Rao PV, Shastry BA. Azithromycin as treatment for cryptosporidiosis in human immunodeficiency virus disease. J Postgrad Med, 2002;48(3):179.

Kirubakaran SI. Update: cytomegalovirus infection in HIVinfected patients-a review. Clin Microbiol Newsl, 2004;26(18):137-44.

Kreuter A, Wieland U. Oral hairy leukoplakia: a clinical indicator of immunosuppression. CMAJ, 2011;183(8):932.

Kumarasamy N, Patel A, Pujar S. Antiretroviral therapy in Indian setting: When \& what to start with, when \& what to switch to? Indian J Med Res, 2011;134(6):787-800.

Lawn SD, Torok ME, Wood R. Optimum time to start antiretroviral therapy during HIV-associated opportunistic infections. Curr Opin Infect Dis, 2011;24(1):34.

Madi D, Achappa B, Rao S, Ramapuram JT, Mahalingam S. Successful treatment of cerebral toxoplasmosis with clindamycin: a case report. Oman Med J, 2012;27(5):411-2.

Mandal BK. Herpes zoster in the immunocompromized populations. Indian J Dermatol, 2006;51(4):235-43.

Mofenson LM, Oleske J, Serchuck L, Van Dyke R, Wilfert C. Treating opportunistic infections among HIV-exposed and infected children- CDC. MMWR Recomm Rep, 2004;53(14):1-63.

NACO -Annual Report 2013-14. [ONLINE] Available from: www.naco.gov.in/upload/.../NACO_English\%202013-14.pdf [Accessed 20 November 2015]

NACO- 2007. Guidelines for Prevention and Management of Common Opportunistic Infections/Malignancies among HIV-infected Adult and Adolescent[ONLINE] Available from: naco.gov.in/...\%20Guidelines/7Guidelines\%20for\%20Prevention\%20an [Accessed 20 November 2015]

NACO- May 2013. Antiretroviral Therapy Guidelines for HIV Infected Adults and Adolescents. [ONLINE] Available from: www.naco.gov.in/...\%20Guidelines/Antiretrovira1\%20Therapy\%20Guide.. . [Accessed 21 November 2015]

NACO -November 2006. Guidelines for HIV Care and Treatment in Infants and Children. [ONLINE] Available from: pps.who.int/medicinedocs/documents/s18022en/s18022en.pdf [Accessed 24 November 2015

Nelson M, Dockrell DH, Edwards S. British HIV Association and British Infection Association guidelines for the treatment of opportunistic infection in HIV- seropositive individuals. HIVmedicine, 2011;12(2):1-5

Njei B, Kongnyuy EJ, Kumar S, Okwen MP, Sankar MJ, Mbuagbaw L. Optimal timing for antiretroviral therapy initiation in patients with HIV infection and concurrent cryptococcal meningitis. Cochrane Database Syst Rev, 2013; 2:CD009012.

Pappas PG, Rex JH, Sobel JD, Filler SG, Dismukes WE, Walsh $\mathrm{TJ}$, et al. Guidelines for treatment of candidiasis. Clin Infect Dis, 2004:38(2):161-89.

Park K. Epidemiology of communicable diseases- AIDS. Test book of preventive and social medicine. $23^{\text {rd }}$ ed. Jabalpur: Banarsidas Bhanot publishers; 2015. p. 344.

Patil HV, Patil VC, Rajmane V, Raje V. Successful treatment of cerebral toxoplasmosis with cotrimoxazole. Indian J Sexual Transm Dis AIDS, 2011;32(1):44.

Pham PA, Flexner C. Emerging antiretroviral drug interactions. J Antimicrob Chemother, 2010;66(2):235-9.

Phillips P, Chan K, Hogg R, Bessuille E, Black W, Talbot J, et al. Azithromycin prophylaxis for Mycobacterium avium complex during the era of highly active antiretroviral therapy: evaluation of a provincial program. Clin InfectDis, 2002;34(3):371-8.

Saadatnia G, Golkar M. A review on human toxoplasmosis. Scand J infect Dis, 2012;44(11):805-14

Saag MS, Graybill RJ, Larsen RA, Pappas PG, Perfect JR, Powderly WG, et al. Practice guidelines for the management of cryptococcal disease. Clin Infect Dis, 2000;30(4):710-8.

Saha K, Firdaus R, Santra P, Pal J, Roy A, Bhattacharya MK, et al. Recent pattern of Co-infection amongst HIV seropositive individuals in tertiary care hospital, Kolkata. Virol J, 2011;8:116-24. 
Seddon J, Bhagani S. Antimicrobial therapy for the treatment of opportunistic infections in HIV/AIDS patients: a critical appraisal. HIV/AIDS (Auckl), 2011;3:19-33.9

Sharma SK, Soneja M. HIV \& immune reconstitution inflammatory syndrome (IRIS). Indian J Med Res, 2011;134(6):866-77.

Strick LB, Wald A, Celum C. Management of herpes simplex virus type 2 infection in HIV type 1-infected persons. Clin Infect Dis, 2006;43(3):347-56.

Swami A, Thakuria R, Kharat S. Cerebral toxoplasmosis in a treatment naive HIV patient with high CD4 count responding to treatment with a regime of cotrimoxazole and pyrimethamine: do we need to start prophylaxis for toxoplasmosis at a higher CD4 count. HIV/AIDS and African American women: research opportunities to stem the epidemic, 2015;2(3):72-5.

Tan IL, Smith BR, von Geldern G, Mateen FJ, McArthur JC. HIV-associated opportunistic infections of the CNS. Lancet Neurol, 2012;11(7):605-17.

UNAids-2014, The Gap Report. [ONLINE] Available from: www.unaids.org/.../unaids/.../unaidspublication/2014/UNAIDS_Gap_rep... [Accessed 20 November 2015]

Vilar FJ, Khoo SH, Walley T. The management of Pneumocystis carinii pneumonia. Br J clin pharmacol, 1999;47(6):605-10.
Walker NF, Scriven J, Meintjes G, Wilkinson RJ. Immune reconstitution inflammatory syndrome in HIV-infected patients. HIV/AIDS (Auck), 2015;7:49-64.

Walubo A. The role of cytochrome P450 in antiretroviral drug interactions. Expert Opin Drug Metab Toxicol, 2007; 3(4): 583-98.

Wazir JF, Ansari NA. Pneumocystis carinii infection. Arch Pathol Lab Med, 2004;128(9):1023-7.

Wilkin A, Feinberg J. Pneumocytis Carinii Pneumonia: A Clinical Review. Am Fam Physician, 1999; 60(6): 1699-1708.

\section{How to cite this article:}

Holla SN, Kamal Kishore MK, Amberkar MB. Pharmacotherapy Of Opportunistic Infections In HIV Patients: A Review. J App Pharm Sci, 2016; 6 (09): 227-236. 